\title{
Hepatocelluar nodules in liver cirrhosis: hemodynamic evaluation (angiography- assisted CT) with special reference to multi-step hepatocarcinogenesis
}

\author{
Osamu Matsui, Satoshi Kobayashi, Junichiro Sanada, Wataru Kouda, Yasuji Ryu, \\ Kazuto Kozaka, Azusa Kitao, Koichi Nakamura, Toshifumi Gabata \\ Department of Radiology, Graduate School of Medical Science, Kanazawa University, Kanazawa, Japan
}

\begin{abstract}
To understand the hemodynamics of hepatocellular carcinoma (HCC) is important for the precise imaging diagnosis and treatment, because there is an intense correlation between their hemodynamics and pathophysiology. Angiogenesis such as sinusoidal capillarization and unpaired arteries shows gradual increase during multi-step hepatocarcinogenesis from high-grade dysplastic nodule to classic hypervascular HCC. In accordance with this angiogenesis, the intranodular portal supply is decreased, whereas the intranodular arterial supply is first decreased during the early stage of hepatocarcinogenesis and then increased in parallel with increasing grade of malignancy of the nodules. On the other hand, the main drainage vessels of hepatocellular nodules change from hepatic veins to hepatic sinusoids and then to portal veins during multi-step hepatocarcinogenesis, mainly due to disappearance of the hepatic veins from the nodules. Therefore, in early HCC, no perinodular corona enhancement is seen on portal to equilibrium phase $\mathrm{CT}$, but it is definite in hypervascular classical HCC. Corona enhancement is thicker in encapsulated HCC and thin in HCC without pseudocapsule. To understand these hemodynamic changes during multi-step hepatocarcinogenesis is important, especially for early diagnosis and treatment of HCCs.
\end{abstract}

Correspondence to: Osamu Matsui; email: matsuio@med.kanazawau.ac.jp
Key words: Hepatocellular carcinoma-Blood supply-Multi-step hepatocarcinogenesis-Early HCC - Dysplastic nodule - Liver

Hepatocellular carcinoma (HCC) is the most common primary liver cancer worldwide. Approximately $80 \%$ of Japanese $\mathrm{HCC}$ cases are derived from HCV-associated liver cirrhosis and chronic hepatitis, and the remaining less than $20 \%$ of the patients are HBV positive. The patients with hepatitis B or C cirrhosis are especially classified as a very high-risk group. Ultrasonography is performed every 3-4 months for the very high-risk group. Because of the introduction of this surveillance system, the size of HCCs firstly detected during 2002$2003(n=33731)$ was less than $2 \mathrm{~cm}$ in $32.5 \%$ of all cases, $2.1-5.0 \mathrm{~cm} 47.0 \%$, respectively [1]. However, various types of hepatocellular nodules such as dysplastic nodule $(\mathrm{DN})$ are also detected during screening procedures. Ultrasound and CT features of DNs and early HCCs are similar, and a precise differential diagnosis is impossible. Pathologically, human HCC develops in a multistep fashion from DN to classic hypervascular HCC. Therefore, for the early diagnosis of HCC, understanding of the concept of multi-step hepatocarcinogenesis and the sequential changes of imaging findings in accordance with multi-step hepatocarcinogenesis is important.

To understand the hemodynamics of HCC is important for the precise imaging diagnosis and treatment, because there is an intense correlation between its hemodynamic and pathophysiology. For this purpose, dynamic MDCT is most valuable because of its high 
spatial and contrast resolution. However, because of the dual blood supply of the liver and intravenous injection of the contrast medium, the precise analysis of hemodynamics by conventional MDCT is often difficult. By the introduction of dynamic CT during selective arteriography, including CT during arterial portography (CTAP) [2,3] and CT during hepatic arteriography (CTHA) [4], it has become possible to visualize the distribution of the intra-hepatic portal and arterial blood flow separately with extremely high contrast resolution, and as a result, to analyze precisely the correlation between blood supply and pathophysiology. In this article, blood flow imaging features of HCC will be discussed based on the CTAP and CTHA imaging and pathophysiologic correlations with special reference to multistep hepatocarcinogenesis.

\section{Classification of hepatocellular nodules and multi-step hepatocarcinogenesis}

The concept of multi-step hepatocarcinogenesis and related small hepatocellular nodules in the patients with chronic liver diseases, particularly those with cirrhosis or chronic hepatitis caused by hepatitis $\mathrm{B}$ or $\mathrm{C}$ viruses, was developed mainly in Japan. However, it had not been widely accepted throughout the world and the diagnostic criteria of these nodules different even among the world specialists. However, in 2009, the International Consensus Group for Hepatocellular Neoplasia organized by the world's leading liver pathologists finally reached agreement [5].

According to this report, these nodules are divided into large regenerative nodule, low grade $\mathrm{DN}$ (L-DN), high-grade DN (H-DN), and HCC. In addition, small HCC (less than $2 \mathrm{~cm}$ ) is divided into early $\mathrm{HCC}$ and progressed HCC. Early $\mathrm{HCC}$ has a vaguely nodular appearance and is well differentiated. Progressed HCC has a distinctly nodular pattern and is mostly moderately differentiated, often with evidence of microvascular invasion. L-DNs are vaguely or distinct nodular with mild increase in cell density and no cytologic atypia. H-DNs are more likely to show a vaguely nodular pattern with architectural and/or cytologic atypia, but the atypia is insufficient for a diagnosis of HCC. They show increased cell density, sometimes more than two times higher than the surrounding nontumoral liver, often with an irregular trabecular pattern. Unpaired arteries are found in most lesions, but usually not in great numbers. A nodule with largely H-DN features containing a subnodule of well-differentiated HCC can be seen. Early HCCs are vaguely nodular and are characterized by various combinations of the following major histologic features; (1) increased cell density more than two times that of the surrounding tissue, with an increased nuclear/ cytoplasm ratio and irregular thin-trabecular pattern; (2) varying numbers of portal tracts within the nodule (intratumoral portal tracts); (3) pseudoglandular pattern; (4) diffuse fatty change; and (5) varying numbers of unpaired arteries. Any of the features listed above may be diffused throughout the lesion or may be restricted to an expansile subnodule (nodule-in-nodule). Most importantly, because all of these features may also be found in H-DNs, it is important to note that stromal invasion remains most helpful in differentiating early HCC from H-DNs. However, the application of these criteria is challenging because most histologic criteria are arrayed on a gradual spectrum and cannot be easily summarized as present or absent.

Because of these reasons as described above, it should be realized that there must be various degree of overlaps among imaging features of these nodules and they may show gradual changes during multi-step hepatocarcinogenesis.

\section{Angiogenesis during multi-step hepatocarcinogenesis}

Vascular endothelial growth factor (VEGF) is known to play a critical role in the neovascularization in the development and progression of malignant neoplasms [6, 7]. VEGF is produced by tumor cells, and its binding with VEGF receptors such as Flt-1 and Flk-1, which are expressed on vascular endothelial cells, leads to the proliferation and migration of endothelial cells. In addition, VEGF receptors expressed on tumor cells are involved in tumor proliferation in an autocrine loop via interaction with VEGF produced by the tumor cells themselves [7]. Park et al. [8] reported that the expression of VEGF was correlated with angiogenesis and cell proliferation in hepatocarcinogenesis. On the other hand, tumors often encounter hypoxic conditions during their growth. Under such conditions, hypoxia inducible factor- $1 \alpha(\mathrm{HIF}-1 \alpha)$ promotes the transcriptional activity of angiogenesis-related molecules such as VEGF and erythropoietin by affecting the hypoxia response element and HIF- $1 \alpha$ located in nuclei [7].

We analyzed these changes of the angiogenesis during multi-step hepatocarcinogenesis by immunohistochemical and molecular studies [7]. According to our analysis, it was found that hepatocellular areas around the portal tracts in DNs, including those with sinusoidal capillarization and unpaired arteries, were strongly positive for HIF- $1 \alpha$, whereas this molecule was faintly expressed in the surrounding livers. Cytoplasmic overexpression and intranuclear expression of HIF- $1 \alpha$, a more increased expression pattern, were also observed in $\mathrm{HCC}$, suggesting that cytoplasmic HIF- $1 \alpha$ might have been moved into the nuclei in activated HCC cells. HIF- $1 \alpha$ is involved in the upregulation of genes harboring the hypoxia response element such as VEGF, suggesting that increased expression of HIF- $1 \alpha$ in the areas around the portal tracts of DNs may be responsible for increased expression of 


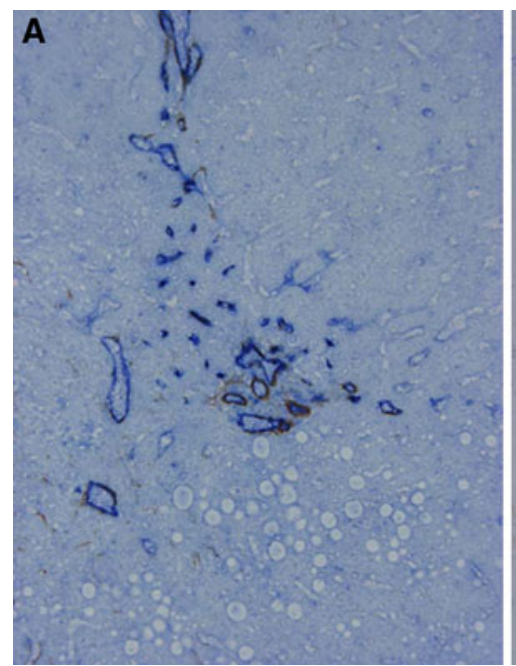

Fig. 1. Angiogenesis during multi-step hepatocarcinogenesis. Sinusoidal capillarization and unpaired arteries are expressed from spotty in $\mathrm{DN}(\mathbf{A}, \times 100)$, diffuse in early hepatocellular carcinoma $(\mathrm{HCC})(\mathbf{B}, \times 50)$ to intense distribution in moderately differentiated $\operatorname{HCC}(\mathbf{C}, \times 100)$ in accor-

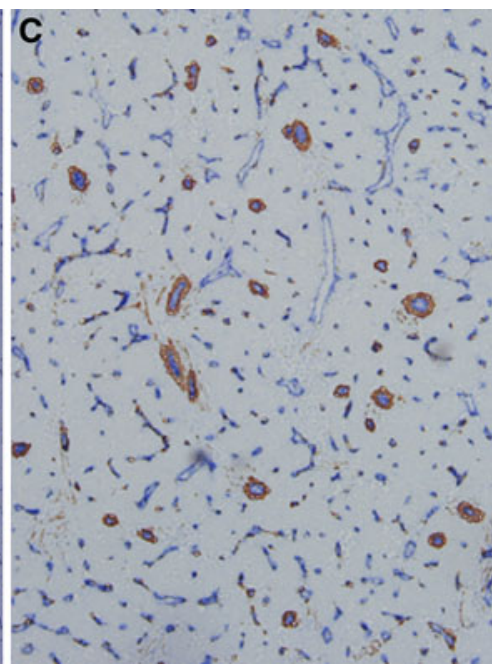

dance with the elevation of the grade of malignancy of the nodules during hepatocarcinogenesis [Double immunohistochemical staining for CD 34 (blue) and $\alpha$-smooth muscle actin (SMA) (brown)].
Fig. 2. Early HCC (highgrade DN with a well-differentiated HCC focus). A CT during hepatic arteriography (CTHA) shows entirely hypodense nodule (arrow $b$ ) with a slightly hyperdense focus (arrow c). B Double immunohistochemical staining for CD34 and $\alpha$ SMA of DN portion (arrow $b$ in A) shows no definite expression of sinusoidal capillarization and unpaired arteries. C Double immunohistochemical staining of $\mathrm{HCC}$ portion corresponding (arrow $c$ in A) shows expression of sinusoidal capillarization and unpaired arteries.
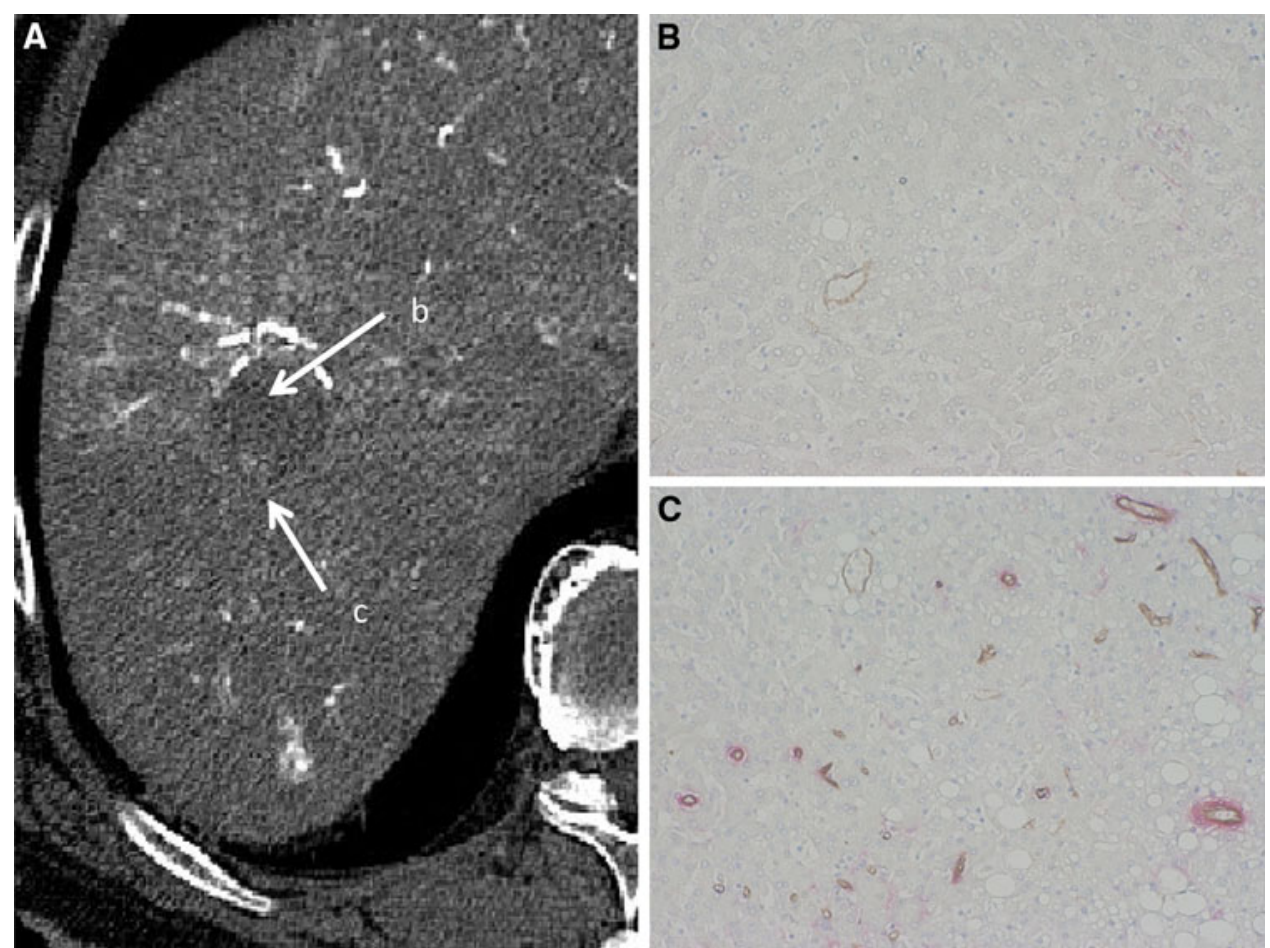

VEGF and its receptor followed by sinusoidal capillarization and increased numbers of unpaired arteries in DNs and also in the angiogenesis in HCC. These expressions gradually spread into the entire nodule in accordance with the elevation of the grade of malignancy of the nodules (Fig. 1).
Figure 2 shows an early HCC consisting of $\mathrm{H}-\mathrm{DN}$ with a small part of highly differentiated HCC with stromal invasion. On CTHA, a well-differentiated focus demonstrates a faint enhancement and this portion reveals more expression of sinusoidal capillarization and unpaired arteries than that in the surrounding H-DN. 
A
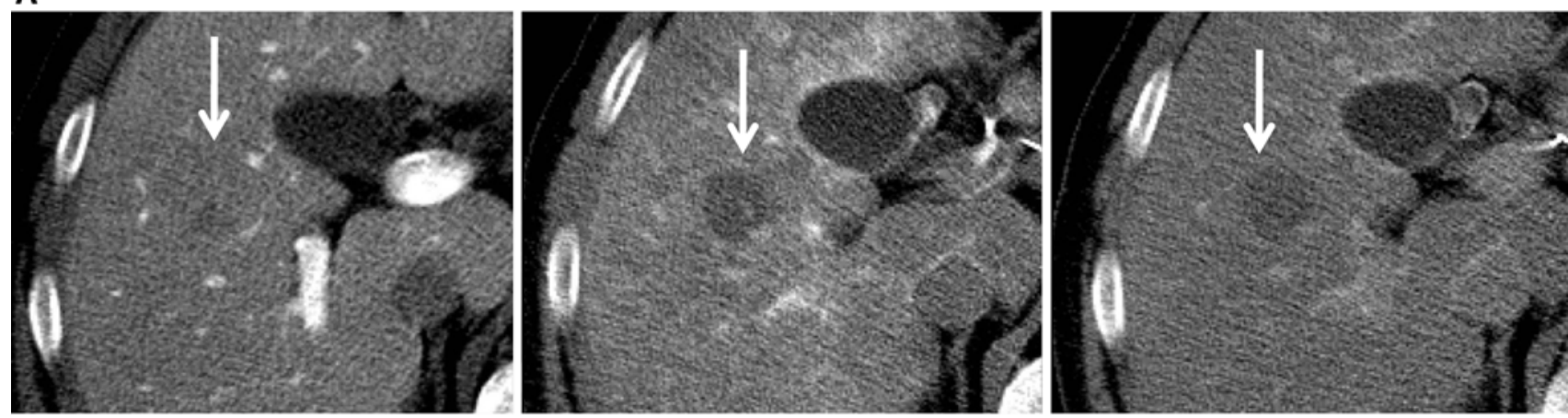

B
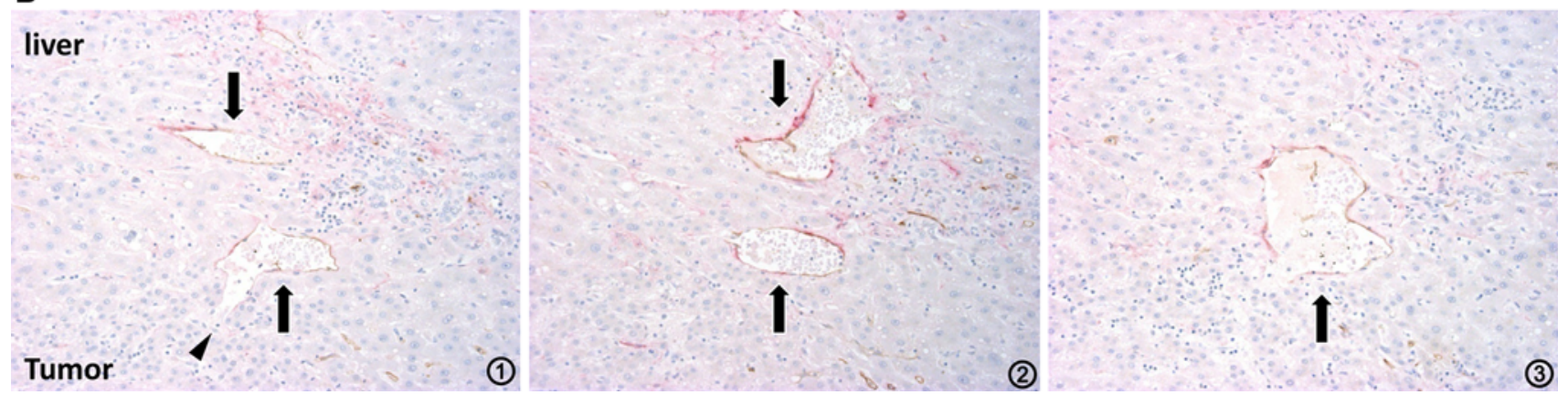

Fig. 3. High-grade DN. A Early phase of CTHA shows a hypodense nodule (middle arrow). It demonstrates isodensity on CT during arterial portography (CTAP, left arrow) and no perilesional enhancement on late phase of CTHA (right arrow).
B Serial specimens from 1 to 3 with double immunohistochemical staining for CD 34 and $\alpha$-SMA show the communication between intratumoral blood sinusoids (arrowhead) and hepatic venules (arrows) in the tumor $(\times 100)$.

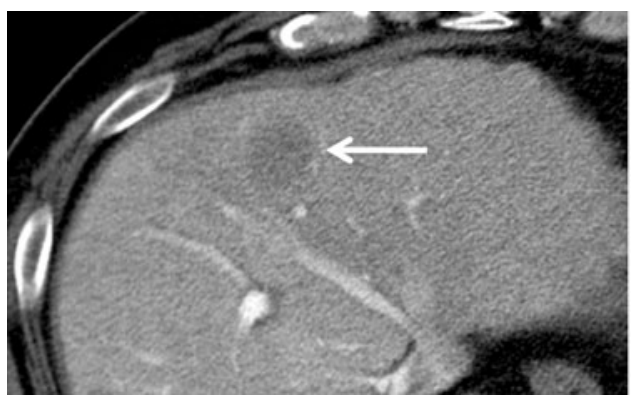

Fig. 4. Early HCC. CTAP (left) shows a faintly hypodense nodule indicating decreased but not absent intranodular portal supply (arrow). On CTHA (right), it is not well identified indi-

This case may indicate the gradual increase of angiogenesis during multi-step hepatocarcinogenesis.

\section{Multi-step changes of intranodular blood supply during hepatocarcinogenesis}

We previously described that the intranodular blood supply evaluated by CTAP and CTHA changed in accordance with hepatocarcinogenesis from DN to overt HCC $[4,9]$. On CTAP, the intranodular portal supply could be divided into four types relative to the sur-

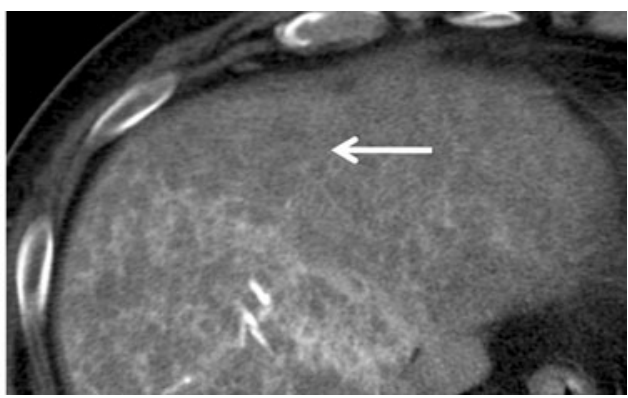

cating almost the same intranodular arterial supply relative to the surrounding liver (arrow).

rounding cirrhotic liver [4] (Figs. 3, 4, 5, 6, 7), namely, isodense nodule relative to the surrounding liver indicating almost the same intranodular portal supply (type A), slightly hypodense nodule indicating decreased but not absent intranodular portal blood flow (type B), a part of the nodule showing a definitely hypodense area indicating a partially absent intranodular portal blood supply (type C) and definitely hypodense indicating an absent intranodular portal supply (type D). The correlation between the histologic types of nodules and these CTAP findings revealed the significant correlation or 


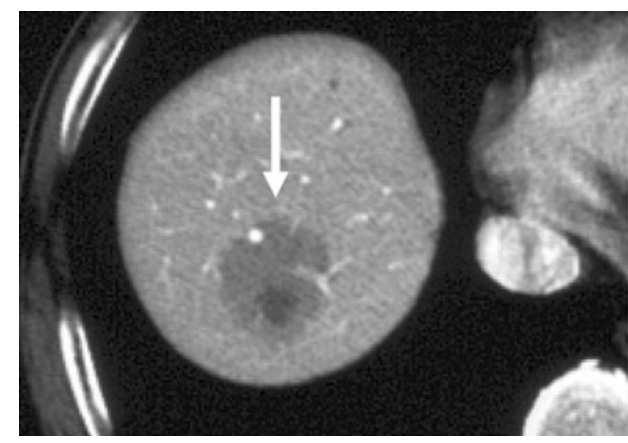

Fig. 5. Well-differentiated HCC (early HCC with subfocus of definite hypervascular well-differentiated HCC). CTAP (left) shows a slightly hypodense nodule with an internal definitely

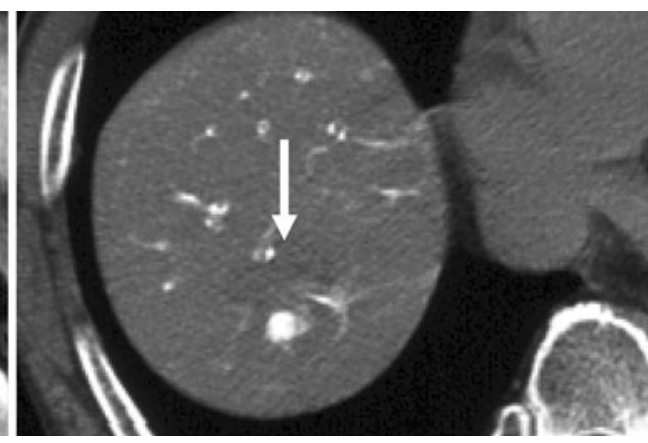

hypodense focus indicating partial portal perfusion defect (arrow). On CTHA (right), it is visualized as an entirely isodense nodule with an internal definitely hypervascular focus (arrow).

A
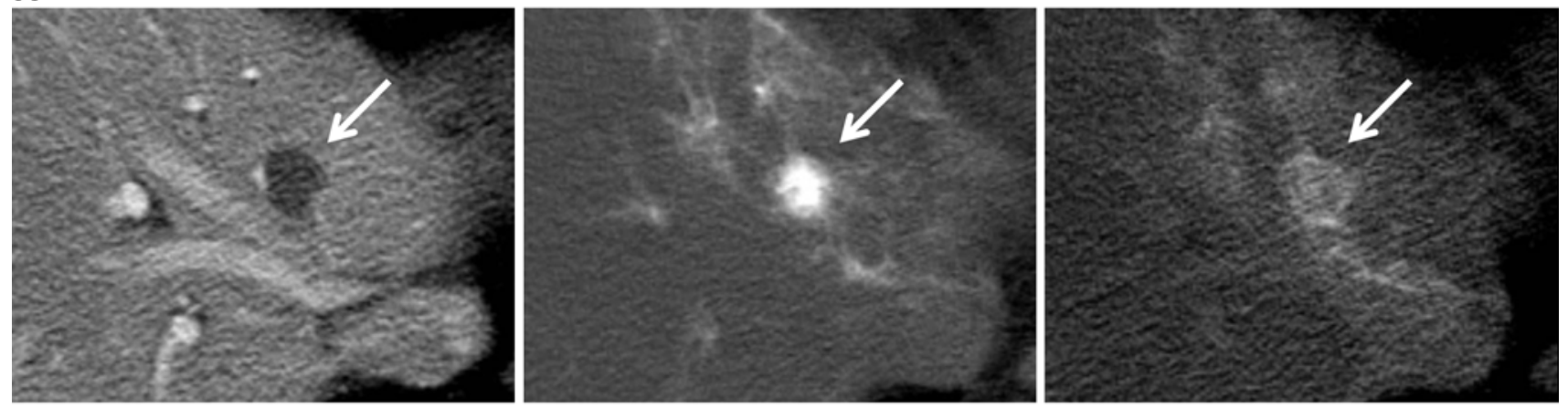

B

Liver (regenerative nodules)

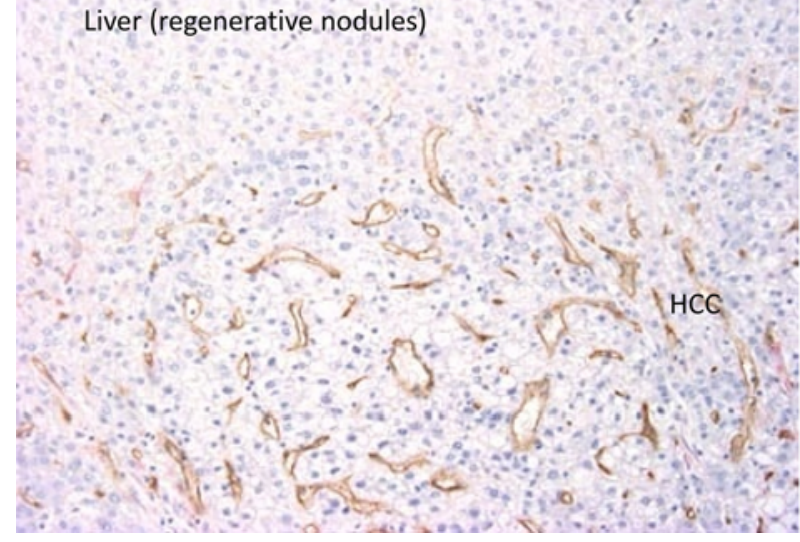

Fig. 6. Hypervascular well-differentiated HCC. A CTAP (left) shows an entirely hypodense nodule (arrow). It demonstrates hyperdensity on early phase of CTHA (middle, arrow) and thin corona enhancement on late phase of CTHA (right, arrow).

strong tendency between DN and type A, early HCC and type B, well-differentiated HCC and type C, and moderately or poorly differentiated HCC and type D (Figs. 3, 4, 5, 6, 7). On CTHA, the intranodular arterial supply could be also categorized into four types relative to the surrounding cirrhotic liver (Figs. 3, 4, 5, 6, 7), namely, isodense nodule indicating almost the same intranodular
B Double immunohistochemical staining of the boundary between the tumor and liver parenchyma shows abundant communications between intratumoral blood sinusoids and hepatic sinusoids $(\times 100)$.

arterial blood supply relative to the surrounding liver (type I), hypodense indicating decreased arterial blood supply (type II), a part of the nodule demonstrating hyperdensity indicating a partially increased arterial supply (type III) and entirely hyperdense indicating entirely increased arterial supply (type IV). The correlation between the histologic types of nodules and these CTHA 
A
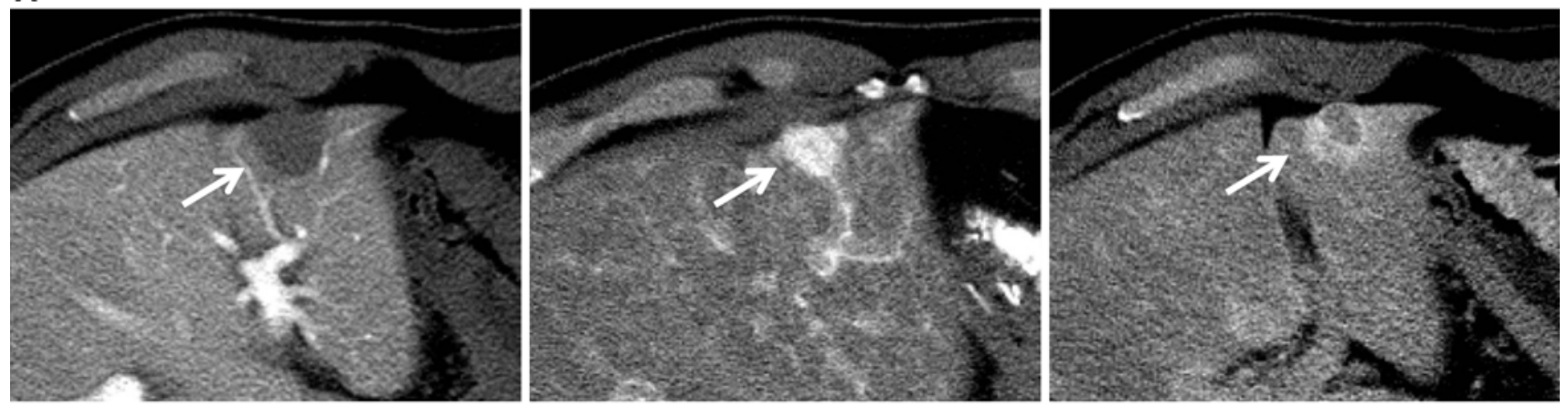

B
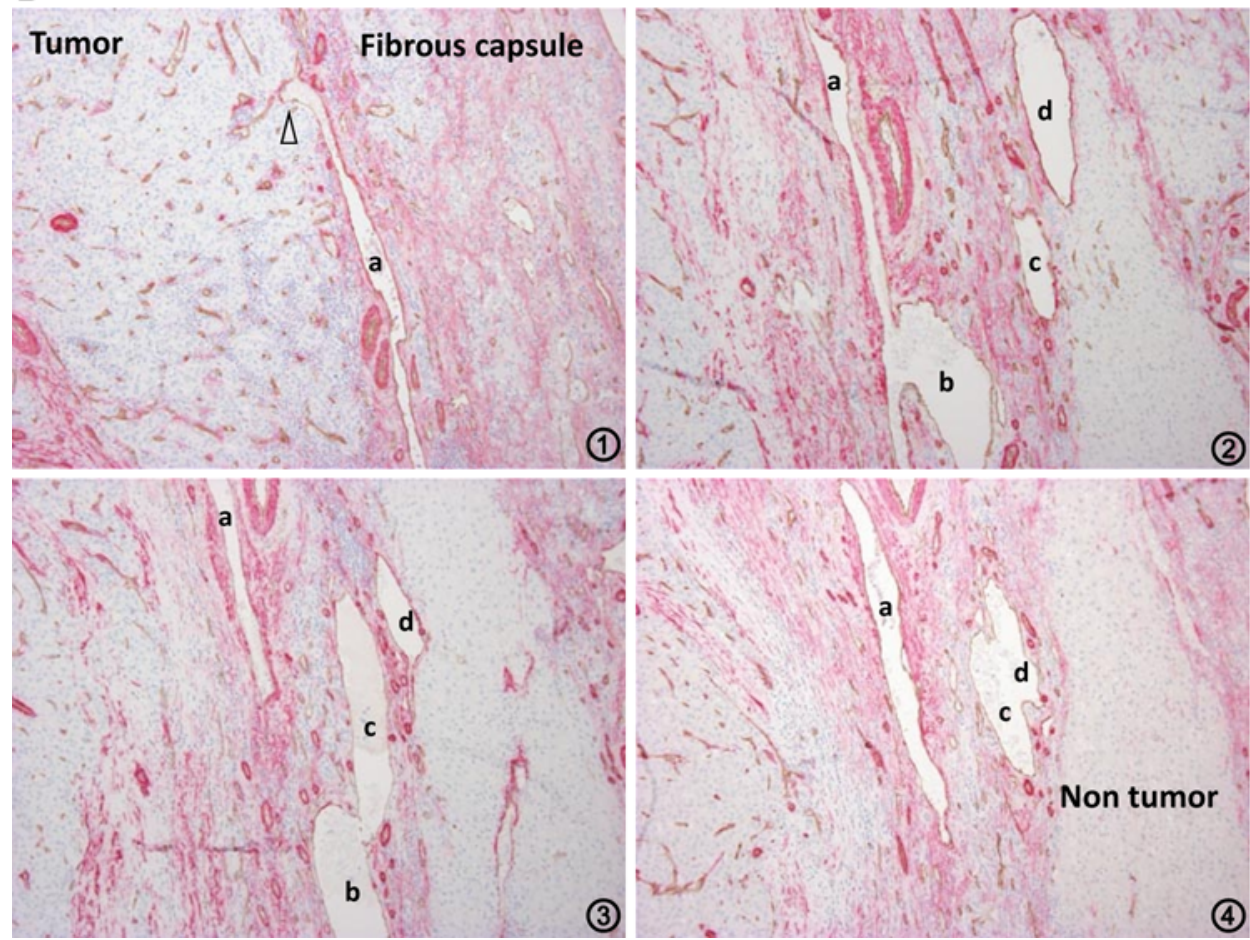

Fig. 7. Moderately differentiated HCC with pseudocapsule formation. A CTAP shows an entirely hypodense nodule (left, arrow). CTHA demonstrates it as an entirely hyperdense nodule on early phase (middle, arrow) with thick corona enhancement on late phase (right, arrow). B Serial specimens

findings revealed the significant correlation or strong tendency between type I and L-DN and early HCC, type II and H-DN and early HCC, type III and well-differentiated HCC and type IV and moderately or poorly differentiated HCC (Figs. 3, 4, 5, 6, 7). These results suggested that the intranodular portal supply relative to the surrounding liver parenchyma is decreased, whereas the intranodular arterial supply is first decreased during the early stage of hepatocarcinogenesis and then increased in parallel with increasing grade of malignancy of the nodules as shown in Fig. 8. However, the differences of histological findings among these nodules are sequential, and the exact diagnosis of the entire nodule is occasionally difficult because of internal histological from 1 to 4 with double immunohistochemical staining for CD34 and $\alpha$ SMA show the communication between intracapsular portal venules $(a-d)$ and intratumoral blood sinusoids (arrowheads) $(\times 100)$.

heterogeneity. Therefore, as shown in the original article [7], there was a fairly wide range of overlap in blood supply patterns among the various types of hepatocellular nodules.

To verify the histological background of the findings obtained by CTAP and CTHA, we analyzed morphometrically the vascular supply of DN and HCCs [10,11], and suggested that the portal tracts including portal vein and hepatic artery were decreased in accordance with increasing grade of malignancy and virtually absent in HCCs. In contrast, abnormal arteries due to tumor angiogenesis developed in $\mathrm{H}-\mathrm{DN}$ during the course of hepatocarcinogenesis, and were markedly increased in number in moderately differentiated HCCs. 
Fig. 8. Multi-step hepatocarcinogenesis and changes of intranodular blood supply. Intranodular portal supply gradually decreases in accordance with the elevation of the grade of malignancy of the nodules and finally disappears in moderately differentiated HCCs. On the other hand, arterial supply first decreases at the early stage of hepatocarcinogenesis and then acutely increases, and finally the entire nodule is fed only by artery in moderately differentiated HCCs.

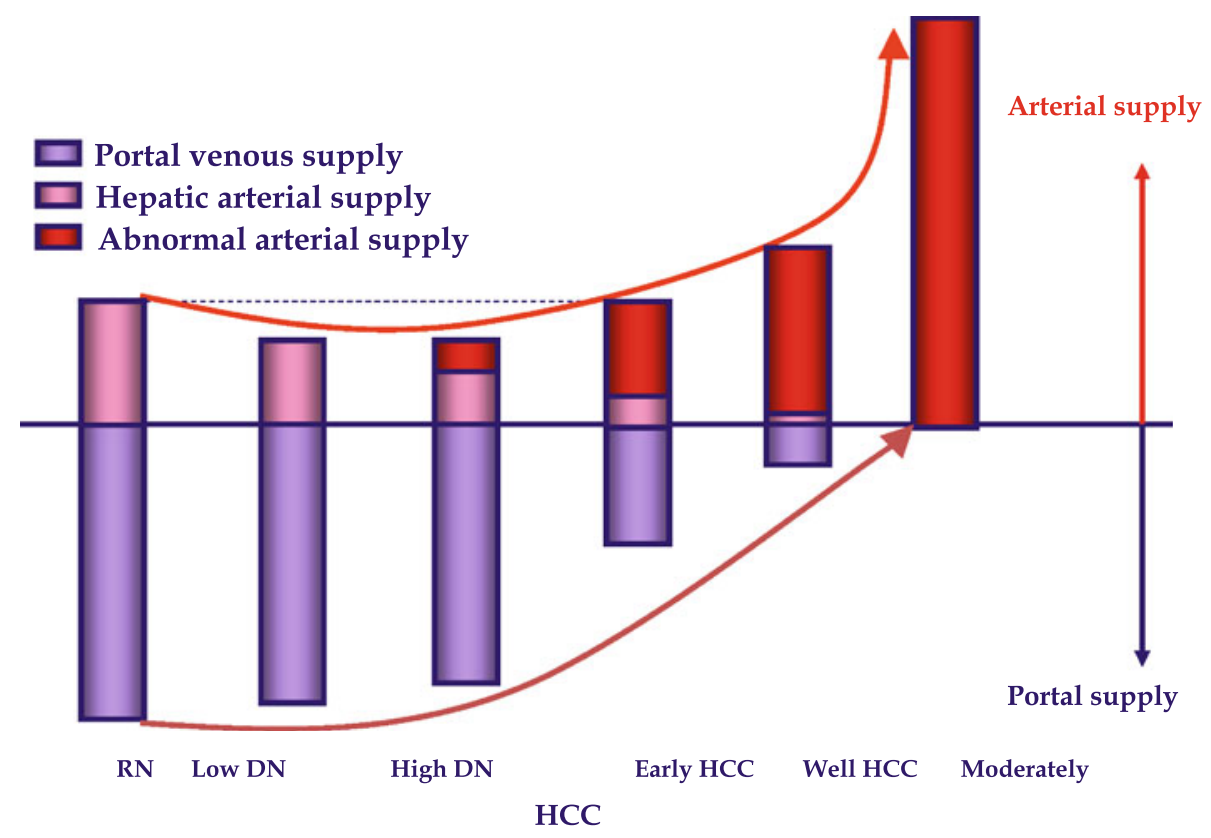

High DN Early HCC Well HCC Moderately HCC

Intranodular and

perinodular

hepatic veins
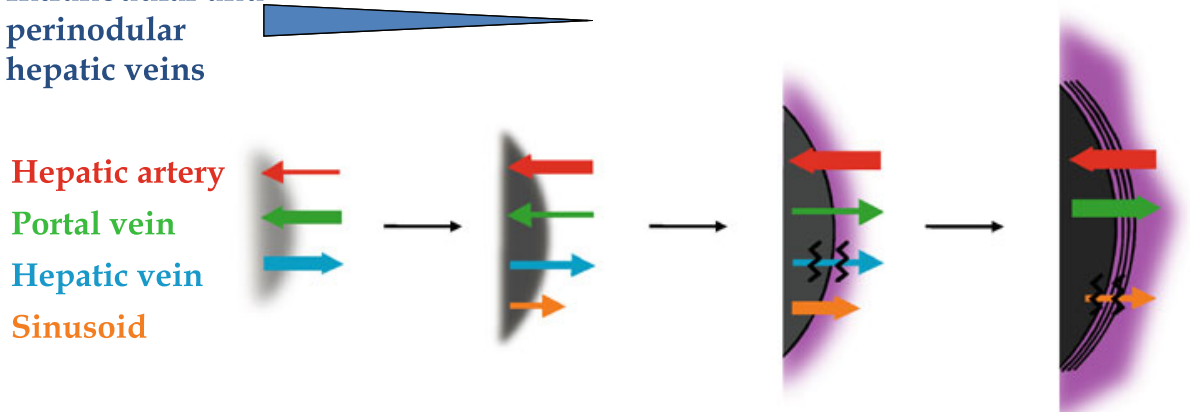

Main drainage vessels

Hepatic vein $\longrightarrow$ Hepatic sinusoids

Portal venules

in capsule and septum
No enhancement
Fig. 9. Multi-step changes of drainage vessels and peritumoral enhancement during hepatocarcinogenesis. In DNs or early HCCs, the main drainage route from the tumor is intranodular or perinodular hepatic vein. However, because hepatic veins disappear from the tumor during very early stage of hepatocarcinogenesis, drainage vessels change to hepatic sinusoids. In moderately differentiated HCC with

\section{Multi-step changes of drainage vessels (flow) during hepatocarcinogenesis}

By single level dynamic thin-section CT during the bolus injection of a small amount of contrast medium, we revealed in vivo hemodynamics in hypervascular classical HCC, namely, the arterial blood flow into the pseudocapsule formation, the communication between tumor sinusoids and the surrounding hepatic sinusoids are also blocked, and then, the portal venules in the pseudocapsule finally become the main drainage vessel from the tumor. In accordance with the changes of the drainage vessels, thin to thick corona enhancement appears surrounding the tumor.

tumor drains into surrounding hepatic sinusoids (corona enhancement) (Fig. 7) [12]. This drainage was well visualized in the late phase of CTHA which was taken after the stoppage of the infusion of the contrast medium into the hepatic artery. Histological examination revealed continuity between a tumor sinusoid and a portal venule in the pseudocapsule (encapsulated HCC) 
or surrounding hepatic sinusoids (HCC without pseudocapsule) $[11,12]$. According to our recent histological study correlated with CTAP and CTHA, the main drainage vessels of hepatocellular nodules change from hepatic veins to hepatic sinusoids and then to portal veins during multi-step hepatocarcinogenesis, mainly due to disappearance of the hepatic veins from the nodules [11]. Therefore, in early HCC, no perinodular corona enhancement is seen on portal to equilibrium phase CT, but it is definite in hypervascular classical HCC (Figs. 3, 6, 7, 9). Corona enhancement is thicker in encapsulated HCC and thin in HCC without pseudocapsule (Figs. 6, 7, 9). The drainage flow from hypervascular HCC variously modified the imaging findings, a feature useful for differential diagnosis. Drainage from the tumor makes the tumor appear larger than it really is on various kinds of blood flow imaging findings. It is the first site of the intrahepatic metastasis of HCC, and daughter nodules are commonly seen in the drainage area. Iodized oil flowed into the surrounding liver through this drainage route and enhanced the effect of transcatheter arterial chemoembolization [13]. The drainage area should be included in RFA area to prevent local recurrence.

\section{Hemodynamics of other hepatic cancers}

Metastatic liver cancers show thin corona enhancement or early peritumoral enhancement on single-level dynamic CTHA [14]. We named the former as "drainage pattern" and the latter as "arterio-portal (AP) shunt pattern". In cases with drainage pattern, the tumor shows hypervascularity in early phase and thin peritumoral enhancement in late phase similar to hypervascular HCC without pseudocapsule, and the drainage route may be the connection between tumor sinusoids and hepatic sinusoids surrounding the tumor $[14,15]$. In cases with AP shunt pattern, the tumor shows no definite enhancement except faint staining on the peripheral margin of the tumor, but early peritumoral enhancement with occasional wedge-shaped expansion is seen. The mechanism of this early enhancement is unknown, but peritumoral multicentric AP shunts due to the obstruction of the portal or hepatic venules can be one of the possible causes. Because abundant fibrous tissue is often contained in this kind of tumor, internal delayed enhancement is commonly associated. Mass-forming type of cholangiocarcinoma usually demonstrates AP shunt pattern. Among malignant primary liver cancer, cholangiolocellular carcinoma (bile ductular carcinoma) shows unique hemodynamics [16]. It typically shows tumor hypervascularity with surrounding enhancement resembling AP shunt pattern in early phase and delayed internal enhancement on late phase, probably due to abundant cancer cells and fibrous tissues in the tumor with multiple entrapped portal tracts in the tumor (replacing infiltration type growth with the portal tracts incorporated into the tumor). Benign hypervascular hepatic masses such as cavernous hemanigoma, focal nodular hyperplasia (FNH) [17], angiomyolipoma and peliosis hepatis usually do not show corona enhancement, probably due to main drainage to hepatic vein. Two exceptions are hepatic adenoma and hypervascular hyperplastic nodule associated with alcoholic cirrhotic livers which commonly demonstrate corona enhancement [18]. Understanding these hemodynamic differences among various kinds of hepatic mass lesions are important for differential diagnosis and treatment.

In conclusion, it is very important to know the hemodynamics of HCCs and related hepatocellular nodules for the understanding of pathophysiology and precise imaging diagnosis and treatment of HCCs. For this purpose, angiography-assisted CT is most valuable and accurate, but because of its invasiveness, blood flow imaging with contrast ultrasound, dynamic CT and MR imaging is necessary.

Open Access. This article is distributed under the terms of the Creative Commons Attribution Noncommercial License which permits any noncommercial use, distribution, and reproduction in any medium, provided the original author(s) and source are credited.

\section{References}

1. Ikai I, Arii S, Okazaki M, et al. (2007) Report of the 17th nationwide follow-up survey of primary liver cancer in Japan. Hepatol Res 37:676-691

2. Matsui O, Kadoya M, Suzuki M, et al. (1983) Work in progress: dynamic sequential computed tomography during arterial portography in the detection of hepatic neoplasms. Radiology 146:721727

3. Matsui O, Takashima T, Kadoya M, et al. (1985) Dynamic computed tomography during arterial portography: the most sensitive examination for small hepatocellular carcinomas. J Comput Assist Tomogr 9:19-24

4. Hayashi M, Matsui O, Ueda K, et al. (1999) Correlation between the blood supply and grade of malignancy of hepatocellular nodules associated with liver cirrhosis: evaluation by CT during intraarterial injection of contrast medium. AJR Am J Roentgenol 172:969-976

5. International Consensus Group for Hepatocellular Neoplasia (2009) Pathologic diagnosis of early hepatocellular carcinoma: a report of the international consensus group for hepatocellular neoplasia. Hepatology 49:658-664

6. Ferrara N (2004) Vascular endothelial growth factor: basic science and clinical progress. Endocr Rev 25:581-611

7. Nakamura K, Zen Y, Sato Y, et al. (2007) Vascular endothelial growth factor, its receptor Flk-1, and hypoxia inducible factorlalpha are involved in malignant transformation in dysplastic nodules of the liver. Hum Pathol 38:1532-1546

8. Park YN, Kim YB, Yang KM, et al. (2000) Increased expression of vascular endothelial growth factor and angiogenesis in the early stage of multistep hepatocarcinogenesis. Arch Pathol Lab Med 124:1061-1065

9. Matsui O, Kadoya M, Kameyama T, et al. (1991) Benign and malignant nodules in cirrhotic livers: distinction based on blood supply. Radiology 178:493-497

10. Ueda K, Terada T, Nakanuma Y, Matsui O (1992) Vascular supply in adenomatous hyperplasia of the liver and hepatocellular carcinoma: a morphometric study. Hum Pathol 23:619-626 
11. Kitao A, Zen Y, Matsui O, Gabata T, Nakanuma Y (2009) Hepatocarcinogenesis: multistep changes of drainage vessels at CT during arterial portography and hepatic arteriography-radiologicpathologic correlation. Radiology 252:605-614

12. Ueda K, Matsui O, Kawamori Y, et al. (1998) Hypervascular hepatocellular carcinoma: evaluation of hemodynamics with dynamic CT during hepatic arteriography. Radiology 206:161-166

13. Terayama N, Matsui O, Gabata T, et al. (2001) Accumulation of iodized oil within the nonneoplastic liver adjacent to hepatocellular carcinoma via the drainage routes of the tumor after transcatheter arterial embolization. Cardiovasc Intervent Radiol 24:383-387

14. Terayama N, Matsui O, Ueda K, et al. (2002) Peritumoral rim enhancement of liver metastasis: hemodynamics observed on singlelevel dynamic CT during hepatic arteriography and histopathologic correlation. J Comput Assist Tomogr 26:975-980
15. Liu Y, Matsui O (2007) Changes of intratumoral microvessels and blood perfusion during establishment of hepatic metastases in mice. Radiology 243:386-395

16. Kozaka K, Matsui O, Nakanuma Y, et al. (2007) A subgroup of intrahepatic cholangiocarcinoma with an infiltrating replacement growth pattern and a resemblance to reactive proliferating bile ductules: 'bile ductular carcinoma'. Histopathology 51:390-400

17. Miyayama S, Matsui O, Ueda K, et al. (2000) Hemodynamics of small hepatic focal nodular hyperplasia: evaluation with single-level dynamic CT during hepatic arteriography. AJR Am J Roentgenol 174:1567-1569

18. Kobayashi S, Matsui O, Kamura T, et al. (2007) Imaging of benign hypervascular hepatocellular nodules in alcoholic liver cirrhosis: differentiation from hypervascular hepatocellular carcinoma. J Comput Assist Tomogr 31:557-563 